\title{
BOUNDED APPROXIMATION BY POLYNOMIALS WHOSE ZEROS LIE ON A CIRCLE $\left({ }^{1}\right)$
}

\author{
BY \\ CHARLES KAM-TAI CHUI
}

1. Introduction. Let $C$ be a rectifiable Jordan curve with interior $D$. We say that a sequence of polynomials $P_{n}$ converges boundedly to a function $f$ in $D$, or $f$ is boundedly approximated by $P_{n}$ in $D$, if $P_{n}$ converges to $f$ throughout $D$ and $\sup \left\{\left|P_{n}(z)\right|: z \in D\right\}$ is bounded as a function of $n$. A polynomial whose zeros lie on $C$ will be called a $C$-polynomial. It is obvious that the limit function of a boundedly convergent sequence of $C$-polynomials in $D$ is a bounded zero free holomorphic function in $D$, unless it is identically zero. In this paper, we will present a proof of the somewhat unexpected converse for the case when $C$ is a circle, as announced in [1].

MAIN THEOREM. Every bounded zero free holomorphic function in the open unit disc can be boundedly approximated there by polynomials whose zeros lie on the unit circumference.

More generally, suppose that $C$ is any rectifiable Jordan curve, so smooth that its parametric representation in terms of arc length has a Hölder continuous derivative. The methods developed in this paper can be extended and modified to prove the possibility of bounded approximation by $C$-polynomials of functions $f$, defined and zero free in clos $D$, such that the derivative $f^{\prime}$, relative to clos $D$, exists and is Hölder continuous throughout clos $D$. In particular, any function $f$ holomorphic and zero free on clos $D$ can be boundedly approximated by $C$ polynomials [1], [2]. However, for arbitrary Jordan curves $C$, the problem of bounded approximation by $C$-polynomials is open, even when $f$ is holomorphic and zero free in clos $D$.

It should be mentioned that a weaker kind of approximation by $C$-polynomials was studied by G. R. MacLane [7]. He proved that if $C$ is a rectifiable Jordan curve with interior $D$ and $f$ is holomorphic and zero free in $D$, then there exists a sequence of $C$-polynomials which converges to $f$ uniformly on every compact subset of $D$. This result was later extended by J. Korevaar and his students [5], [6], [8] to other domains $D$. Very recently, Professor Korevaar and the author considered the case where $C$ is the disjoint union of two or more Jordan curves [3]. It is interesting to note how the approximation problem by $C$-polynomials breaks down in some situations.

Received by the editors November 9, 1967 and, in revised form, April 19, 1968.

( ${ }^{1}$ ) This work was partially supported by NSF grant GP-4106. It is part of the author's doctoral dissertation at the University of Wisconsin written under Professor Jacob Korevaar. 
Throughout the rest of this paper, $C$ will denote the unit circle and $D$, the open unit disc.

2. Approximation of $S_{k}(z)=\prod_{m=1}^{k}\left(1-z e^{-i \theta_{m}}\right)^{\alpha_{m}}$. We first construct a sequence of $C$-polynomials $P_{n}$ which converges to the function

$$
S_{k}(z)=\prod_{m=1}^{k}\left(1-z e^{-i \theta_{m}}\right)^{\alpha_{m}}
$$

in $D$, where $0 \leqq \theta_{1}<\cdots<\theta_{k}<2 \pi$ and $0 \leqq \alpha_{m}<1, m=1, \ldots, k$.

The proof of the uniform boundedness of $P_{n}$ on $D$ will be included in $\S 4$. We use the same construction as indicated in [1]. Set $\sum_{m=1}^{k} \alpha_{m}=\alpha$. For all $n$ so large that $2 \pi /(n+k-\alpha)<\min \left(\theta_{m+1}-\theta_{m}\right)$, where $\theta_{k+1}=2 \pi+\theta_{1}$, we define numbers $t_{j}=t_{j}(n)$ and $\theta_{m}^{*}(n), j=1, \ldots, n$ and $m=1, \ldots, k$, by the following procedure

$$
t_{1}=\theta_{1}+\frac{\left(2-\alpha_{1}\right) \pi}{n+k-\alpha}, t_{2}=t_{1}+\frac{2 \pi}{n+k-\alpha}, \cdots, t_{j_{1}}=t_{j_{1}-1}+\frac{2 \pi}{n+k-\alpha},
$$

where $j_{1}$ is determined by the inequality

$$
\begin{gathered}
t_{j_{1}}<\theta_{2} \leqq t_{j_{1}}+\frac{2 \pi}{n+k-\alpha}, \cdots, t_{j_{1}+1}=t_{j_{1}}+\frac{2\left(2-\alpha_{2}\right)}{n+k-\alpha}, \\
t_{j_{1}+2}=t_{j_{1}+1}+\frac{2 \pi}{n+k-\alpha}, \cdots, t_{j_{2}}=t_{j_{2}-1}+\frac{2 \pi}{n+k-\alpha},
\end{gathered}
$$

where $j_{2}$ is determined by the inequality $t_{j_{2}}<\theta_{3} \leqq t_{j_{2}}+2 \pi /(n+k-\alpha)$;

$$
\begin{aligned}
t_{j_{k-1}+1} & =t_{j_{k-1}}+2\left(2-\alpha_{k}\right) \pi /(n+k-\alpha), \\
t_{j_{k-1}+2} & =t_{j_{k-1}+1}+2 \pi /(n+k-\alpha), \ldots, \\
t_{j_{k}} & =t_{j_{k-1}}+2 \pi /(n+k-\alpha) ;
\end{aligned}
$$

here $j_{k}$ is determined by the inequality

$$
t_{j_{k}}<\theta_{1}+2 \pi \leqq t_{j_{k}}+2 \pi /(n+k-\alpha)
$$

finally,

$$
\theta_{1}^{*}=\theta_{1}, \quad \theta_{l}^{*}=\frac{1}{2}\left(t_{j_{l-1}}+t_{j_{l-1}+1}\right), \quad l=2, \ldots, k
$$

Hence,

$$
\left|\theta_{m}-\theta_{m}^{*}\right| \leqq \frac{\left(2-\alpha_{m}\right) \pi}{n+k-\alpha}<\frac{2 \pi}{n} \text { for } m=1, \ldots, k
$$

We now define

$$
P_{n+k}(z, k)=\prod_{j=1}^{n}\left(1-z e^{-i t_{j}}\right) \prod_{m=1}^{k}\left(1-z e^{-i \theta_{m}^{*}}\right) .
$$


We shall prove

TheOREM 2.1. For every $k$ and every $z \in D, P_{n+k}(z, k) \rightarrow S_{k}(z)$, as $n \rightarrow \infty$.

To prove this, we need a lemma of the Riemann-Lebesgue type. For each $n$, let $s_{n}$ be the following step function on $[0,2 \pi]$. The jumps of $s_{n}$ only occur at $t_{1}, \ldots, t_{n}$ and $\theta_{1}^{*}, \ldots, \theta_{k}^{*}$. Each jump at $t_{j}$ is equal to 1 and each jump at $\theta_{m}^{*}$ is equal to $\left(1-\alpha_{m}\right)$. These conditions do not determine $s_{n}$ completely; however, we find it more convenient to state the additional conditions in terms of the related function,

$$
v_{n}(t)=s_{n}(t)-(n+k-\alpha) t / 2 \pi .
$$

We set $v_{n}\left(\theta_{1}^{*}+\right)=\frac{1}{2}\left(1-\alpha_{1}\right)$ and define $v_{n}\left(\theta_{m}^{*}\right)=v_{n}\left(t_{j}\right)=0$ for $m=1, \ldots, k$ and $j=1, \ldots, n$. Then we have the following

Lemma 2.1. If $g$ is an integrable function on $(0,2 \pi)$, then

$$
\lim _{n \rightarrow \infty} \int_{0}^{2 \pi} g(t) v_{n}(t) d t=0 .
$$

The proof of this lemma is the same as the proof of the Riemann-Lebesgue lemma. We first prove it for the case of a step function, and then approximate $g$ by step functions, cf. [9].

Now we can prove Theorem 2.1. For fixed $z \in D$, let $g$ be the integrable function

$$
g(t)=\frac{d}{d t} \log \left(1-z e^{-i t}\right)=\frac{d}{d t}\left\{-\sum_{p=1}^{\infty} \frac{z^{p}}{p} e^{-i p t}\right\}
$$

Here, as everywhere else in this paper, we choose the principal values of the logarithms. Integration by parts gives

$$
\begin{aligned}
\int_{0}^{2 \pi} g(t) v_{n}(t) d t & =\int_{0}^{2 \pi}-\log \left(1-z e^{-i t}\right) d v_{n}(t) \\
& =-\sum_{j=1}^{n} \log \left(1-z e^{-i t_{j}}\right)-\sum_{m=1}^{k}\left(1-\alpha_{m}\right) \log \left(1-z e^{-i \theta_{m}^{*}}\right),
\end{aligned}
$$

and this tends to 0 by the lemma. Using (2.2), we see that

$$
\sum_{j=1}^{n} \log \left(1-z e^{-i t_{j}}\right)+\sum_{m=1}^{k} \log \left(1-z e^{-i \theta_{m}^{*}}\right) \rightarrow \sum_{m=1}^{k} \alpha_{m} \log \left(1-z e^{-i \theta_{m}^{*}}\right) .
$$

This is equivalent to $P_{n+k}(z, k) \rightarrow S_{k}(z)$ for each $k$ and each $z \in D$.

3. Bounded approximation by $S_{k}(z)$. The following lemma is a trivial consequence of Herglotz's theorem [4].

LEMMA 3.1. Let $f$ be holomorphic and zero free in $D, f(0)=1$ and $\sup |f(z)|=$ $M<+\infty$. Then there exists a nondecreasing real-valued function $v$ in $[0,2 \pi]$, with $\nu(0)=0$ and $\nu(2 \pi)=2 \log M$, such that

$$
f(z)=\exp \left\{\int_{0}^{2 \pi} \frac{-z e^{-i t}}{1-z e^{-i t}} d \nu(t)\right\} \text { for each } z \text { in } D .
$$


The above lemma indicates the importance of the following function,

$$
f(z)=\exp \{-z /(1-z)\}
$$

which is zero free, holomorphic and bounded in $D$. The first thing we do is to study the bounded approximation of $\exp \{-z /(1-z)\}$ by expressions of the form

$$
S_{k}(z)=\prod_{m=1}^{k}\left(1-z e^{-i 2 \pi m / k}\right)^{\alpha_{k, m}}
$$

throughout $D$, where $\alpha_{k, m} \geqq 0$ for $m=1, \ldots, k$ and

$$
\max _{1 \leqq m \leqq k} \alpha_{k, m} \rightarrow 0, \quad \text { as } k \rightarrow \infty .
$$

We establish the following

TheOREM 3.1. Let $f(z)=\exp \{-z /(1-z)\}$ and $\varepsilon>0$ be given. There exist functions $S_{k}, k=1,2, \ldots$, of the form indicated in (3.2) and (3.3), such that $S_{k}$ converges to $f$ throughout $D$ and

$$
\max _{|z| \leqq 1}\left|S_{k}(z)\right| \leqq e^{1 / 2+\varepsilon} \quad \text { for all } k .
$$

Proof. Let $k$ and $p$ be positive integers with $k>2 p$. We define

$$
\alpha_{k, m}^{(p)}=\sum_{j=1}^{p} \frac{2 j}{k}\left(1-\frac{j}{p}\right)\left(1+\cos \frac{2 \pi j m}{k}\right)
$$

where $m=1, \ldots, k$. Clearly, each $\alpha_{k, m}^{(p)} \geqq 0$ and

$$
\max _{1 \leqq m \leqq k} \alpha_{k, m}^{(p)} \leqq \frac{p^{2}}{k} .
$$

For each $z$ in $D$,

$$
\begin{aligned}
\sum_{m=1}^{k}(1 & \left.+\cos \frac{2 \pi j m}{k}\right) \log \left(1-z e^{-i 2 \pi m / k}\right) \\
= & -\sum_{v=1}^{\infty} \sum_{m=1}^{k} \frac{z^{v}}{\nu} e^{-i 2 \pi v m / k}\left(1+\cos \frac{2 \pi j m}{k}\right) \\
= & -\sum_{v=1}^{\infty} \frac{z^{\nu}}{\nu}\left\{\sum_{m=1}^{k} e^{-i 2 \pi v m / k}+\sum_{m=1}^{k} \cos \frac{2 \pi v m}{k} \cdot \cos \frac{2 \pi j m}{k}\right. \\
& \left.-i \sum_{m=1}^{k} \sin \frac{2 \pi v m}{k} \cdot \cos \frac{2 \pi j m}{k}\right\}
\end{aligned}
$$

Here,

$$
\begin{aligned}
\sum_{m=1}^{k} e^{-i 2 \pi v m / k} & =k & & \text { if } k \mid \nu \\
& =0 & & \text { if } k \nmid \nu .
\end{aligned}
$$


It follows that

$$
\begin{aligned}
& \sum_{m=1}^{k} \cos \frac{2 \pi \nu m}{k} \cdot \cos \frac{2 \pi j m}{k}=\frac{1}{2} \sum_{m=1}^{k}\left\{\cos \frac{2 \pi(\nu+j) m}{k}+\cos \frac{2 \pi(\nu-j) m}{k}\right\} \\
& =(1 / 2) k \text { if } k \mid(\nu+j) \text { or } k \mid(\nu-j) \text {, } \\
& =0 \quad \text { otherwise. }
\end{aligned}
$$

( $k$ cannot divide both $\nu+j$ and $\nu-j$ since $2 j \leqq 2 p \leqq k$.) Similarly,

$$
\sum_{m=1}^{k} \sin \frac{2 \pi \nu m}{k} \cos \frac{2 \pi j m}{k}=\frac{1}{2} \sum_{m=1}^{k}\left\{\sin \frac{2 \pi(\nu+j) m}{k}+\sin \frac{2 \pi(\nu-j) m}{k}\right\}=0 .
$$

Note that $k \mid(\nu+j)$ if and only if $\nu=\mu k-j$ where $\mu=1,2, \ldots$ and $k \mid(\nu-j)$ if and only if $\nu=\mu k+j$ where $\mu=0,1,2, \ldots$ Hence, substituting (3.8), (3.9) and (3.10) into (3.7) and combining with (3.5), we get

$$
\begin{aligned}
\sum_{m=1}^{k} \alpha_{k, m}^{(p)} \log (1 & \left.-z e^{-i 2 \pi m / k}\right)=-\sum_{j=1}^{p} \frac{2 j}{k}\left(1-\frac{j}{p}\right) \\
& \times\left\{\frac{k}{2} \cdot \frac{z^{j}}{j}+\sum_{\mu=1}^{\infty} \frac{z^{\mu k}}{\mu k} k+\sum_{\mu=1}^{\infty} \frac{z^{\mu k+j}}{\mu k+j} \cdot \frac{k}{2}+\sum_{\mu=1}^{\infty} \frac{z^{\mu k-j}}{\mu k-j} \cdot \frac{k}{2}\right\} .
\end{aligned}
$$

To estimate this expression, we compare the terms with denominators $\mu k \pm j$ with similar terms with denominators $\mu k$. For each $z$ in $D$, we get, remembering $p<\frac{1}{2} k$,

$$
\begin{aligned}
\mid \sum_{j=1}^{p} \frac{2 j}{k}\left(1-\frac{j}{p}\right)\left\{\sum_{\mu=1}^{\infty} \frac{z^{\mu k \pm j}}{\mu k \pm j}\right. & \left.\cdot \frac{k}{2}-\sum_{\mu=1}^{\infty} \frac{z^{\mu k}}{\mu k} \cdot \frac{k}{2}\right\} \mid \\
& \leqq \sum_{j=1}^{p} j\left(1-\frac{j}{p}\right) \sum_{\mu=1}^{\infty} \frac{j}{(\mu k-j) \mu k} \\
& \leqq \sum_{j=1}^{p} j^{2}\left(1-\frac{j}{p}\right) \sum_{\mu=1}^{\infty} \frac{2}{\mu^{2} k^{2}}<\frac{\pi^{2}}{36} \cdot \frac{p^{3}}{k^{2}}
\end{aligned}
$$

Hence, uniformly for $z \in D$,

$$
\begin{aligned}
\sum_{m=1}^{k} \alpha_{k, m}^{(p)} \log \left(1-z e^{-i 2 \pi m / k}\right)= & -\sum_{j=1}^{p}\left(1-\frac{j}{p}\right) z^{j}+\sum_{j=1}^{p} \frac{2 j}{k}\left(1-\frac{j}{p}\right) \\
& \times\left\{-\sum_{\mu=1}^{\infty} \frac{z^{\mu k}}{\mu}-\frac{z^{j}}{2} \sum_{\mu=1}^{\infty} \frac{z^{\mu k}}{\mu}-\frac{z^{-j}}{2} \sum_{\mu=1}^{\infty} \frac{z^{\mu k}}{\mu}\right\}+O\left(p^{3} / k^{2}\right) \\
= & -\sum_{j=1}^{p}\left(1-\frac{j}{p}\right) z^{j}+\sum_{j=1}^{p} \frac{2 j}{k}\left(1-\frac{j}{p}\right) \\
& \times\left(1+\frac{z^{j}+z^{-j}}{2}\right) \log \left(1-z^{k}\right)+O\left(p^{3} / k^{2}\right) .
\end{aligned}
$$


By the maximum principle for harmonic functions,

$$
\begin{aligned}
& \max _{|z| \leq 1} \sum_{m=1}^{k} \alpha_{k, m}^{(p)} \log \left|1-z e^{-i 2 \pi m / k}\right| \\
& \leqq \max _{0 \leqq \theta \leqq 2 \pi}\left\{\sum_{j=1}^{p}-\left(1-\frac{j}{p}\right) \cos j \theta\right\} \\
&+\max _{0 \leqq \theta \leqq 2 \pi}\left\{\sum_{j=1}^{p} \frac{2 j}{k}\left(1-\frac{j}{p}\right)(1+\cos j \theta) \log \left|1-e^{i k \theta}\right|\right\} \\
&+O\left(p^{3} / k^{2}\right)=I_{1}+I_{2}+O\left(p^{3} / k^{2}\right),
\end{aligned}
$$

say. Since $\log \left|1-e^{i k \theta}\right| \leqq \log 2$,

$$
I_{2} \leqq 4 \log 2 \sum_{j=1}^{p} \frac{j}{k}\left(1-\frac{j}{p}\right)<\frac{p^{2}}{k} .
$$

Also, it is well known that

$$
-\sum_{j=1}^{p}\left(1-\frac{j}{p}\right) \cos j \theta=\frac{1}{2}-F_{p}(\theta)
$$

when $F_{p}(\theta)$ denotes the Fejér kernel of order $p$. Since the Fejér kernels are nonnegative, we see that $I_{1} \leqq \frac{1}{2}$ for all $p$. Hence,

$$
\max _{|z| \leqq 1} \prod_{m=1}^{k}\left|1-z e^{-i 2 \pi m / k}\right|_{k, m}^{\alpha_{k, m}^{(p)}} \leqq \exp \left[\frac{1}{2}+O\left(p^{2} / k\right)\right] .
$$

Furthermore, from (3.12) we see that for each $z \in D$,

$$
\sum_{m=1}^{k} \alpha_{k, m}^{(p)} \log \left(1-z e^{-i 2 \pi m / k}\right)=\sum_{j=1}^{p}-\left(1-\frac{j}{p}\right) z^{j}+O\left(\frac{p^{2}}{k}|z|^{k-p}\right)+O\left(\frac{p^{3}}{k^{2}}\right) .
$$

We now let $k$ tend to $\infty$ through the positive integers, and let $p$ tend to $\infty$ in such a way that $p^{2} / k \rightarrow 0$. The series $-\sum_{j=1}^{\infty} z^{j}$ converges to $-z /(1-z)$ in $D$; it follows that the Cesàro means

$$
-\sum_{j=1}^{p}\left(1-\frac{j}{p}\right) z^{j}
$$

of the partial sums also converge to $-z /(1-z)$. Thus for $z \in D$,

$$
\sum_{m=1}^{k} \alpha_{k, m}^{(p)} \log \left(1-z e^{-i 2 \pi m / k}\right)
$$

converges to $-z /(1-z)$. We conclude, by (3.6), that from the numbers $\alpha_{k, m}^{(p)}$, we can form a family of finite sequences $\left\{\alpha_{k, m}\right\}, m=1, \ldots, k, k=1,2, \ldots$ which satisfies the conditions of the theorem. 
We are now ready to prove a more general result, namely

THEOREM 3.2. Let $f$ be any zero free holomorphic function in $D, f(0)=1$ and $\sup |f(z)|=M<+\infty$. Then for any $\varepsilon>0$, there exists a sequence of functions $S_{k}$ of the form indicated in (3.2) and (3.3), such that $S_{k}$ converges to $f$ throughout $D$, and

$$
\max _{|z| \leqq 1}\left|S_{k}(z)\right| \leqq M^{1+\varepsilon} \quad \text { for all } k \text {. }
$$

Proof. By Lemma 3.1, we can find a nondecreasing real-valued function $v(t)$ on $[0,2 \pi]$ with $\nu(0)=0, \nu(2 \pi)=2 \log M$ such that

$$
f(z)=\exp \left\{\int_{0}^{2 \pi} \frac{-z e^{-i t}}{1-z e^{-i t}} d \nu(t)\right\}
$$

for $z \in D$. Thus, setting

$$
\beta_{n, j}=\nu(2 \pi j / n)-\nu(2 \pi(j-1) / n),
$$

where $j=1, \ldots, n$, so that $\beta_{n, j} \geqq 0$ and $\sum_{j=1}^{n} \beta_{n, j}=2 \log M$, we have

$$
f(z)=\lim _{n \rightarrow \infty} f_{n}(z)
$$

where

$$
f_{n}(z)=\prod_{j=1}^{n} \exp \left\{\beta_{n, j} \frac{-z e^{-i 2 \pi j / n}}{1-z e^{-i 2 \pi j / n}}\right\}
$$

It is clear that

$$
\sup _{|z|<1}\left|f_{n}(z)\right| \leqq M
$$

for all $n$. By Theorem 3.1, we can choose finite sequences $\gamma_{m, p}, p=1, \ldots, m$, $m=1,2, \ldots$, such that $\gamma_{m, p} \geqq 0$ and $\max _{p} \gamma_{m, p} \rightarrow 0$,

for $w \in D$ and

$$
S_{m}(w)=\prod_{p=1}^{m}\left(1-w e^{-i 2 \pi p / m}\right)^{\gamma_{m, p}} \rightarrow \exp \left\{\frac{-w}{1-w}\right\}
$$

$$
\max _{|w| \leqq 1}\left|S_{m}(w)\right| \leqq e^{(1+\varepsilon) / 2}
$$

for all $m$. In particular, for fixed integers $n, j$ and $z \in D$, the sequence

$$
S_{m}\left(z e^{-i 2 \pi j / n}\right)^{\beta}, \mathrm{J}
$$

converges to

$$
\exp \left\{-\beta_{n, j} z e^{-i 2 \pi j / n} /\left(1-z e^{-i 2 \pi j / n}\right)\right\} .
$$

We now take our integers $m$ of the form $k n, k=1,2, \ldots$, and define

$$
S_{k}(z, n)=\prod_{j=1}^{n} S_{k n}\left(z e^{-i 2 \pi j / n}\right)^{\beta} n, j=\prod_{p=1}^{n}\left(1-z e^{-i 2 \pi p / n}\right)^{\alpha_{k n, p}}
$$

where

$$
\alpha_{k n, p}=\sum_{j+q=p(\bmod k n)} \gamma_{k n, q} \beta_{n, j}
$$


for $p=1, \ldots, k n$. Clearly, $\alpha_{k n, p} \geqq 0$ and

$$
\max _{p} \alpha_{k n, p} \leqq \sum_{j=1}^{n} \beta_{n, j} \cdot \max _{q} \gamma_{k n, q}=2 \log M \cdot \max _{q} \gamma_{k n, q},
$$

which tends to 0 as $k$ tends to $\infty$. Furthermore, for fixed $n$ and $z \in D$,

$$
S_{k}(z, n) \rightarrow f_{n}(z), \quad \text { as } k \rightarrow \infty
$$

and for all $k$, and all $n$,

$$
\max _{|z| \leqq 1}\left|S_{k}(z, n)\right| \leqq \prod_{j=1}^{n}\left\{e^{(1+\varepsilon) / 2}\right\}^{\beta_{n, j}}=M^{1+\varepsilon} .
$$

Now let $A_{K}=\{z:|z| \leqq 1-1 / K\}$. By (3.14) and the Stieltjes-Osgood theorem, $f_{n}$ converges uniformly to $f$ on $A_{K}$; and by (3.15), and fixed $n, S_{k}(z, n)$ converges uniformly to $f_{n}$. Hence, for a fixed integer $K$, we determine $n=n_{K}$ so large that

$$
\max _{z \in A_{K}}\left|f_{n}(z)-f(z)\right|<\frac{1}{K}
$$

and determine $k=k_{K}$ so large that for $n=n_{K}$

$$
\max _{z \in A_{\underline{B}}}\left|S_{k}(z, n)-f_{n}(z)\right|<\frac{1}{K}
$$

Setting $S_{K}(z)=S_{k_{K}}\left(z, n_{K}\right)$, we complete the proof of the theorem.

4. Bounded approximation by $C$-polynomials. We observe the following

LEMMA 4.1. For $0<\alpha \leqq \pi / 4$ and $|z|<1$, we have

$$
\log |1-z|-\frac{1}{2 \alpha} \int_{-\alpha}^{\alpha} \log \left|1-z e^{-i t}\right| d t<3 .
$$

Proof. By the maximum principle it is sufficient to consider $z=e^{i \theta}$, and by symmetry, we assume that $0<\theta \leqq \pi$. We first note that

$$
\begin{aligned}
\log \left|1-e^{i \theta}\right|-\frac{1}{2 \alpha} \int_{-\alpha}^{\alpha} \log \left|1-e^{i(\theta-t)}\right| d t & =\log \sin \frac{\theta}{2}-\frac{1}{2 \alpha} \int_{-\alpha}^{\alpha} \log \left|\sin \frac{\theta-t}{2}\right| d t \\
& \leqq \frac{1}{2 \alpha} \int_{-\alpha}^{\alpha} \log \left|\frac{\theta}{\theta-t}\right| d t+2 \\
& =\frac{\theta}{2 \alpha} \int_{-\alpha / \theta}^{\alpha / \theta} \log \frac{1}{|1-u|} d u+2 .
\end{aligned}
$$

The function

$$
h(x)=\frac{1}{2 x} \int_{-x}^{x} \log \frac{1}{|1-u|} d u
$$

is continuous on $(0, \infty), h(0+)=0$, and $h(x) \rightarrow-\infty$ as $x \rightarrow+\infty$. Hence, $h(x)$ must be bounded above on $(0, \infty)$. Actually, it is bounded above by 1 . This completes the proof of the lemma. 
LEMMA 4.2. For each $m=2,3, \ldots$, let $\tau_{k}=\tau_{k}(m), k=1, \ldots, m$, be points on $[0,2 \pi]$ such that $0<\tau_{1}<\cdots<\tau_{m} \leqq 2 \pi$, and, setting $\tau_{m+1}=\tau_{1}+2 \pi$,

$$
\max _{1 \leqq k \leqq m}\left(\tau_{k+1}-\tau_{k}\right) / \min _{1 \leqq k \leqq m}\left(\tau_{k+1}-\tau_{k}\right) \leqq A .
$$

Also let $\omega_{k}=\omega_{k}(m)$ and $\alpha_{k}=\alpha_{k}(m)$ be defined as

$$
\omega_{k}=\frac{1}{2}\left(\tau_{k+1}+\tau_{k}\right), \quad \alpha_{k}=m\left(\tau_{k+1}-\tau_{k}\right) / 2 \pi .
$$

Finally, let

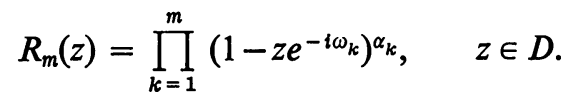

Then for all large $m$,

$$
\max _{|z| \leqq 1}\left|R_{m}(z)\right| \leqq \exp \left(19 A^{3}\right) .
$$

Proof. For convenience, set $\frac{1}{2}\left(\tau_{k+1}-\tau_{k}\right)=\Delta_{k}=\Delta_{k}(m)$, and let $n_{1}$ denote the positive integer $\left[\sqrt{ } 2 A^{2}\right]+2$. Without loss of generality, we can assume that the maximum of $\left|R_{m}(z)\right|$ is attained at $e^{i \theta}$, where $\tau_{m}-2 \pi \leqq \theta \leqq \tau_{1}$. Since

we may write

$$
\int_{0}^{2 \pi} \log \left|1-e^{i t}\right| d t=0
$$

$$
\log \left|R_{m}\left(e^{i \theta}\right)\right|=\sum_{k=1}^{m} \frac{m}{2 \pi} \int_{-\Delta_{k}}^{\Delta_{k}} \log \left|\frac{1-e^{i\left(\theta-\omega_{k}\right)}}{1-e^{i\left(\theta-\omega_{k}-t\right)}}\right| d t .
$$

We split the sum into two parts $\Sigma^{\prime}+\Sigma^{\prime \prime}$, where $\Sigma^{\prime}$ denotes the sum over $1 \leqq k \leqq n_{1}-1$ and $m-n_{1}+1 \leqq k \leqq m, \Sigma^{\prime \prime}$ denotes the sum over $n_{1} \leqq k \leqq m-n_{1}$.

By Lemma 4.1, we can conclude that for all large $m$ (those for which $\left.\Delta_{k}(m) \leqq \pi / 4\right)$,

$$
\Sigma^{\prime}=\sum^{\prime} \alpha_{k}\left\{\frac{1}{2 \Delta_{k}} \int_{-\Delta_{k}}^{\Delta_{k}} \log \left|\frac{1-e^{i\left(\theta-\omega_{k}\right)}}{1-e^{i\left(\theta-\omega_{k}-t\right)}}\right| d t\right\} \leqq 3 \sum^{\prime} \alpha_{k} .
$$

By (4.2), we see that

$$
\min _{1 \leqq k \leqq m}\left(\tau_{k+1}-\tau_{k}\right) \geqq \frac{1}{A} \cdot \frac{2 \pi}{m} ; \quad \max _{1 \leqq k \leqq m}\left(\tau_{k+1}-\tau_{k}\right) \leqq A \cdot \frac{2 \pi}{m} .
$$

Hence, since $A \geqq 1$,

$$
\begin{aligned}
\Sigma^{\prime} & \leqq 3 \cdot \max \alpha_{k} \cdot \text { number of terms in } \Sigma^{\prime} \\
& \leqq 3 \cdot \frac{m}{\pi} A \frac{2 \pi}{m}\left(2 n_{1}-1\right)<18 A^{3} .
\end{aligned}
$$

We now turn to $\Sigma^{\prime \prime}$. We can write

$$
\begin{aligned}
\sum^{\prime \prime} & =\frac{m}{2 \pi} \sum_{k=n_{1}}^{m-n_{1}} \int_{0}^{\Delta_{k}} \log \left|\frac{\left(1-e^{i\left(\theta-\omega_{k}\right)}\right)^{2}}{\left(1-e^{i\left(\theta-\omega_{k}\right)}\right)\left(1-e^{i\left(\theta-\omega_{k}+t\right)}\right)}\right| d t \\
& =\frac{m}{2 \pi} \sum_{k=n_{1}}^{m-n_{1}} \int_{0}^{\Delta_{k}}-\log \frac{\left(1-\cos \left(\theta-\omega_{k}-t\right)\right)^{1 / 2}\left(1-\cos \left(\theta-\omega_{k}+t\right)\right)^{1 / 2}}{1-\cos \left(\theta-\omega_{k}\right)} d t .
\end{aligned}
$$


Using the trigonometric identity

$$
(1-\cos (\alpha-\beta))(1-\cos (\alpha+\beta))=(\cos \alpha-\cos \beta)^{2},
$$

we obtain

$$
\begin{aligned}
\sum^{\prime \prime} & =\frac{m}{2 \pi} \sum_{k=n_{1}}^{m-n_{1}} \int_{0}^{\Delta_{k}}-\log \left(1-\frac{1-\cos t}{1-\cos \left(\theta-\omega_{k}\right)}\right) d t \\
& =\frac{m}{2 \pi} \sum_{k=n_{1}}^{m-n_{1}} \int_{0}^{\Delta_{k}}-\log \left(1-\frac{\sin ^{2}(t / 2)}{\sin ^{2}\left(\left(\theta-\omega_{k}\right) / 2\right)}\right) d t .
\end{aligned}
$$

Setting

$$
\begin{aligned}
& B_{1}=\left\{k: n_{1} \leqq k \leqq m-n_{1}, 0 \leqq \omega_{k}-\theta \leqq \pi\right\} \\
& B_{2}=\left\{k: n_{1} \leqq k \leqq n-n_{1}, \pi \leqq \omega_{k}-\theta \leqq 2 \pi\right\}
\end{aligned}
$$

we see that for $k \in B_{1}$,

$$
\sin \left(\left(\omega_{k}-\theta\right) / 2\right) \geqq\left(\omega_{k}-\theta\right) / \pi,
$$

and for $k \in B_{2}$,

$$
\sin \left(\left(\omega_{k}-\theta\right) / 2\right) \geqq 2-\left(\omega_{k}-\theta\right) / \pi=\left[(2 \pi+\theta)-\omega_{k}\right] / \pi .
$$

Hence, for $0 \leqq t \leqq \Delta_{k}$ and $k \in B_{1}$,

$$
\begin{aligned}
0 & \leqq \frac{\sin ^{2}(t / 2)}{\sin ^{2}\left(\left(\theta-\omega_{k}\right) / 2\right)} \leqq \frac{\left(\Delta_{k} / 2\right)^{2}}{\left[\left(\omega_{k}-\theta\right) / \pi\right]^{2}} \leqq \frac{\pi^{2}}{4} \frac{\Delta_{k}^{2}}{\left(\tau_{k}-\tau_{1}\right)^{2}} \\
& \leqq \frac{\pi^{2}}{4^{2}} \cdot \frac{A^{4}}{\left(n_{1}-1\right)^{2}}<\frac{1}{2},
\end{aligned}
$$

by the definition of $n_{1}$. The same inequality holds for $0 \leqq t \leqq \Delta_{k}$ and $k \in B_{2}$. Thus by the inequality

$$
-\log (1-x) \leqq x /(1-x) \leqq 2 x, \text { for } 0 \leqq x \leqq \frac{1}{2},
$$

and by (4.6), we get

$$
\begin{aligned}
\sum^{\prime \prime} \leqq & \frac{m}{\pi} \sum_{k=n_{1}}^{m} \int_{0}^{n_{1}} \frac{\sin ^{2}(t / 2)}{\sin ^{2}\left(\left(\theta-\theta_{k}\right) / 2\right)} d t \\
\leqq & \frac{m}{\pi} \sum_{k \in B_{1}} \int_{0}^{\Delta_{k}} \frac{\pi^{2}}{4\left(\omega_{k}-\theta\right)^{2}} t^{2} d t \\
& +\frac{m}{\pi} \sum_{k \in B_{2}} \int_{0}^{\Delta_{k}} \frac{\pi^{2}}{4\left[(2 \pi+\theta)-\omega_{k}\right]^{2}} t^{2} d t \\
\leqq & \frac{\pi}{12} m \sum_{k \in B_{1}} \frac{\Delta_{k}^{3}}{\left(\tau_{k}-\tau_{1}\right)^{2}}+\frac{\pi}{12} m \sum_{k \in B_{2}} \frac{\Delta_{k}^{3}}{\left(\tau_{m}-\tau_{k+1}\right)^{2}}<A^{3} .
\end{aligned}
$$

Combining this with (4.5), we obtain (4.4). 
Now we have enough machinery to prove the main theorem, namely

THEOREM 4.1. Let $f$ be any zero free holomorphic function in $D, f(0)=1$ and $\sup |f|=M<+\infty$. Then there exists a sequence of $C$-polynomials

$$
P_{n}(z)=\prod_{j=1}^{n}\left(1-z e^{-i t_{n, s}}\right)
$$

which converges to $f$ in $D$, and is such that for an arbitrary $\varepsilon>0$,

$$
\max _{|z| \leqq 1}\left|P_{n}(z)\right| \leqq e^{20} \cdot M^{1+\varepsilon} \quad \text { for all } n \text {. }
$$

Proof. By Theorem 3.2, we can find a sequence of functions $S_{k}$ of the form indicated in (3.2) and (3.3) which converges to $f$ in $D$ and is such that for all $k$,

$$
\max _{|z| \leqq 1}\left|S_{k}(z)\right| \leqq M^{1+\varepsilon} .
$$

By (3.3) it may be assumed that for all $k$

$$
\max _{1 \leqq m \leqq k} a_{k, m} \leqq \varepsilon_{1}
$$

where $\varepsilon_{1}$ is an arbitrarily fixed number between 0 and 1 . By $\S 2$, we can find $C$ polynomials

$$
P_{n+k}(z, k)=\prod_{j=1}^{n}\left(1-z e^{-i t_{j}}\right) \prod_{m=1}^{k}\left(1-z e^{-i \theta_{m}^{*}}\right),
$$

taking $\theta_{m}=2 \pi m / k$ in $\S 2$, such that $P_{n+k}$ converges to $S_{k}$ for any fixed $k$.

To get a suitable bound of $P_{n+k}$, we will apply the above lemma, using the present $t_{j}$ and $\theta_{m}^{*}$ as points $\omega_{j}$. The intervals $\left(\tau_{j}, \tau_{j+1}\right)$ in the lemma will have the points $t_{j}$ and $\theta_{m}^{*}$ as midpoints. Thus, the interval $\left(\tau_{j}, \tau_{j+1}\right)$ with midpoint $t_{j}$ will have length $2 \pi /(n+k-\alpha)$; the interval $\left(\tau_{j}, \tau_{j+1}\right)$ with midpoint $\theta_{m}^{*}$ will have length $\left(1-\alpha_{k, m}\right) 2 \pi /(n+k-\alpha)$. Since $p=n+k$, the exponent of $\left(1-z \exp \left(-i t_{j}\right)\right)$ in $R_{p}(z)=$ $R_{n+k}(z)$ will be

$$
\frac{n+k}{2 \pi} \cdot \frac{2 \pi}{n+k-\alpha}=\frac{n+k}{n+k-\alpha}
$$

and the exponent of $\left(1-z e^{-i \theta_{m}^{*}}\right)$ will be

$$
\frac{n+k}{2 \pi}\left(1-\alpha_{k, m}\right) \frac{2 \pi}{n+k-\alpha}=\frac{\left(1-\alpha_{k, m}\right)(n+k)}{n+k-\alpha} \text {. }
$$

It follows that we can take $A=1 /\left(1-\varepsilon_{1}\right)$ in the lemma. Hence, for large $n$,

$$
\max _{|z| \leqq 1}\left|R_{n+k}(z)\right| \leqq \exp \left[19\left(1-\varepsilon_{1}\right)^{-3}\right] \text {. }
$$

That is,

$$
\max _{|z| \leqq 1}\left|P_{n+k}(z, k)\right| \leqq \exp \left[19\left(1-\varepsilon_{1}\right)^{-3}\right] \cdot \max _{|z| \leqq 1} \prod_{m=1}^{k}\left|1-z e^{-i \theta_{m}^{*}}\right|^{\alpha_{k, m}} .
$$


But $\theta_{m}^{*} \rightarrow \theta_{m}=2 \pi m / k$ and the maximum norm is continuous. Hence, for an arbitrary $\varepsilon_{2}>0$, we have, for large $k$,

$$
\max _{|z| \leqq 1} \prod_{m=1}^{k}\left|1-z e^{-i \theta_{m}^{*}}\right|^{\alpha_{k, m}} \leqq\left(1+\varepsilon_{2}\right) \max _{|z| \leqq 1}\left|S_{k}(z)\right| .
$$

Taking $\varepsilon_{1}$ and $\varepsilon_{2}$ sufficiently small, we can conclude that for any $k$ and sufficiently large $n$,

$$
\max _{|z| \leqq 1}\left|P_{n+k}(z, k)\right| \leqq e^{20} M^{1+\varepsilon} .
$$

Since $S_{k}$ also satisfies a uniform boundedness condition (4.8), the same argument as in the proof of Theorem 3.2 implies that there is a sequence of $C$-polynomials

$$
P_{n}(z)=P_{n+k}(z, k), \quad n=n(k),
$$

which satisfies (4.7) and which converges to $f$ throughout $D$.

The bound in (4.7) can be improved just a little if we no longer require that the approximating polynomials have the value 1 at $z=0$. Indeed, let $\varepsilon_{n}$ be a sequence of positive real numbers tending to 0 ; then there is a sequence of $C$-polynomials $P_{m}$ satisfying (4.7) with $\varepsilon$ replaced by $\varepsilon_{n}$. Multiplying $P_{n}$ by $M^{-\varepsilon_{n}}$, we get a sequence of $C$-polynomials $Q_{n}$, which converges to $f$ and satisfies

$$
\max _{|z| \leqq 1}\left|Q_{n}(z)\right| \leqq e^{20} \cdot \sup _{|z|<1}|f(z)| .
$$

That is, we obtain the following

COROLlaRY 4.1. A zero free bounded holomorphic function $f$ in $D$ can be boundedly approximated by C-polynomials $Q_{n}$ in $D$, which satisfy the inequality (4.9) for all $n$.

\section{REFERENCES}

1. C. Chui, Bounded approximation by polynomials with restricted zeros, Bull. Amer. Math. Soc. 73 (1967), 967-972.

2. - Bounded approximation by polynomials with restricted zeros, Ph.D. Thesis, Univ. of Wisconsin, Madison, 1967.

3. J. Korevaar and C. Chui, Potentials of families of unit masses on disjoint Jordan curves, Proc. Conference on Approximation Theory and Abstract Spaces, Oberwolfach, Germany, (to appear).

4. K. Hoffman, Banach spaces of analytic functions, Prentice-Hall, Englewood Cliffs, N. J., 1962, p. 67.

5. J. Korevaar, Asymptotically neutral distributions of electrons and polynomial approximation. Ann. of Math. 80 (1964), 403-410.

6. - "Limits of polynomials whose zeros lie in a given set," in Entire functions and related parts of analysis, (1966 Summer Institute), Proc. Sympos. Pure Math., Vol. 11, Amer. Math. Soc., Providence, R. I., 1968.

7. G. R. MacLane, Polynomials with zeros on a rectifiable Jordan curve, Duke Math. J. 16 (1949), 461-477.

8. M. D. Thompson, Approximation by polynomials whose zeros lie on a curve, Duke Math. J. 31 (1964), 255-265.

9. A. Zygmund, Trigonometric series. I, Cambridge Univ. Press, New York, 1959, p. 49.

STATE UNiversity OF NeW York, Buffalo, New York 